\title{
Maximum Likelihood Identification of Wiener Models with a Linear Regression Initialization
}

\author{
Anna Hagenblad and Lennart Ljung \\ Department of Electrical Engineering \\ Linköping University, S-581 83 Linköping, Sweden \\ WWW: http://www.control.isy.liu.se \\ Email: annah@isy.liu.se
}

August 28, 1998

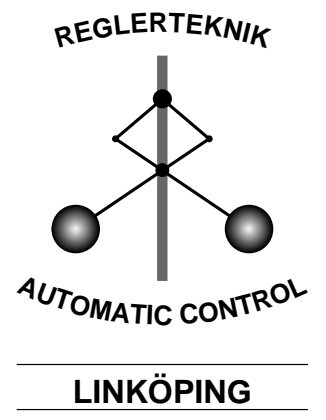

Report no.: LiTH-ISY-R-2051

Submitted to CDC'98

Technical reports from the Automatic Control group in Linköping are available by anonymous ftp at the address ftp.control.isy.liu.se. This report is contained in the compressed postscript file 2051.ps.Z. 


\title{
Maximum Likelihood Identification of Wiener Models with a Linear Regression Initialization
}

\author{
Anna Hagenblad and Lennart Ljung \\ Automatic Control \\ Linköping University \\ S-581 83 Linköping, Sweden \\ email: annah@isy.liu.se, ljung@isy.liu.se
}

\begin{abstract}
Many parametric identification routines suffer from the problem with local minima. This is true also for the prediction-error approach to identifying Wiener models, i.e. linear models with a static non-linearity at the output. We here suggest a linear regression initialization, that secures a consistent and efficient estimate, when used in conjunction with a Gauss-Newton minimization scheme.
\end{abstract}

\section{The Prediction Error Identification Estimate}

A Wiener model consists of a linear dynamic system $x(t)=G(q, \theta) u(t)$ and a static nonlinearity $y(t)=$ $f_{\eta}(x(t))$. Several approaches to the identification of such models have been suggested in the literature. See, e.g., $[5,3,7,1]$. We shall here look into the prediction error/maximum likelihood method, with the criterion numerically minimized by a Gauss-Newton scheme. The difficulty lies in finding an initialization that avoids the problem with local minima. We shall use an idea from [3] to devise such a consistent initial estimate.

We suppose that the nonlinearity is invertible. $G$ and $f$ are parameterized in the parameters $\theta$ and $\eta$, respectively. Assuming white noise at the output of the linear plant (or at the measurement point), the prediction error estimate is found by minimizing (see [4])

$$
\begin{aligned}
V(\theta, \eta) & =\frac{1}{N} \sum_{t=1}^{N} \varepsilon^{2}(t, \theta, \eta)=\frac{1}{N} \sum_{t=1}^{N}(y(t)-\hat{y}(t))^{2} \\
& =\frac{1}{N} \sum_{t=1}^{N}\left(y(t)-f_{\eta}(G(q, \theta) u(t))\right)^{2}
\end{aligned}
$$

This estimate coincides with the maximum-likelihood estimate in case the noise at the measurement point is Gaussian.

For general parameterizations of $G$ and $f$, the criterion (1) cannot be minimized analytically, and we will have to use a numerical search method, like Gauss-Newton. See [2] or [4] for details on the numerical search.

\section{Initialization}

The Gauss-Newton method guarantees convergence to a local minimum of the criterion (1). But in general the criterion has several local minima. It is thus of great importance with a good initialization. We want the initialization to be reliable in that the initial values are close to the global minimum.

The idea is to first parameterize the model as a linear regression with possibly many parameters, so as to guarantee a global minimum of the criterion. This will give a consistent estimate of the linear dynamics, as well as of the static non-linearity. These initial estimates are then transformed to the original parameterization in (1).

Let the linear system $G$ be described by an FIR model

$$
x(t)=c_{1} u(t-1)+\cdots+c_{n} u(t-n)
$$

To get a linear regression estimate of the entire system, we parameterize the inverse of the nonlinear system with linear B-splines

$$
x(t)=d_{1} B_{1}(y(t))+\cdots+d_{m} B_{m}(y(t))
$$

with $m+1$ breakpoints. The selection of the breakpoints is not a trivial issue, and might affect the estimate. One possibility is to space them evenly between the largest and smallest output value, another to distribute them with an equal number of data points around them.

Equating the two sides of the equations gives

$$
\begin{aligned}
& c_{1} u(t-1)+\cdots+c_{n} u(t-n) \\
& =d_{1} B_{1}(y(t))+d_{2} B_{2}(y(t))+\cdots+d_{m} B_{m}(y(t))
\end{aligned}
$$

This is a linear regression that can be solved either by fixing one of its parameters, or by applying the total 
least squares methods (i.e. adding a norm constraint on all of the parameters).

The estimate is consistent, since any stable linear dynamic system can be arbitrarily well approximated by an FIR model by taking $n$ sufficiently large. (The best choice of $n$ will depend on the number of data $N$.) Similarly, the nonlinearity can be described with arbitrary accuracy by taking $m$ sufficiently large.

The FIR model obtained can be converted to another model structure if desired, by model reduction techniques, or by simulating the unmeasurable signal $x$ and estimating a new model, e.g. output-error or state space, from $u$ and $x$ with standard methods. A piecewise linear model of the nonlinearity is immediately obtained from the estimated model of the inverse.

\section{An Example}

As an example, we have used the distillation column data from [1].

By minimizing the prediction error (1) with a GaussNewton numerical search, we got the results shown in Figure 1. The search was initialized as described in section 2. The number of FIR-parameters used were 400 (!, but it turns out that the linear dynamics has a very slowly decaying impulse response), and the number of breakpoints for the nonlinearity 5 . The breakpoints included the maximum and minimum value of the output, and were otherwise distributed to get an even support. $d_{1}$ was fixed to -1000 . From the initial FIR model, a second order ARX model was estimated from $u$ and simulated values of $x$. This second order model was then used in (1). The nonlinearity was parameterized with hinging hyperplanes, see [6]. To measure the quality of the models we have used the prediction error and the variance accounted for (vaf) calculated as

$$
\operatorname{VAF}=\left(1-\frac{\operatorname{var}(\hat{y}(t)-y(t))}{\operatorname{var}(y(t))}\right) \times 100 \%
$$

This value turned out to be 95.0. A straightforward application of the method proposed in [1] (but not estimating initial filter conditions, $x(-1)$ and $x(-2)$, like in the application of (1)) gave a misfit that was twice as big.

Acknowledgement The authors wish to thank Michel Verhaegen for the permission to use the data from the destillation column.

\section{References}

[1] J. Bruls, C. T. Chou, B. R. J Haverkamp, and M. Verhaegen. Linear and non-linear system identi-
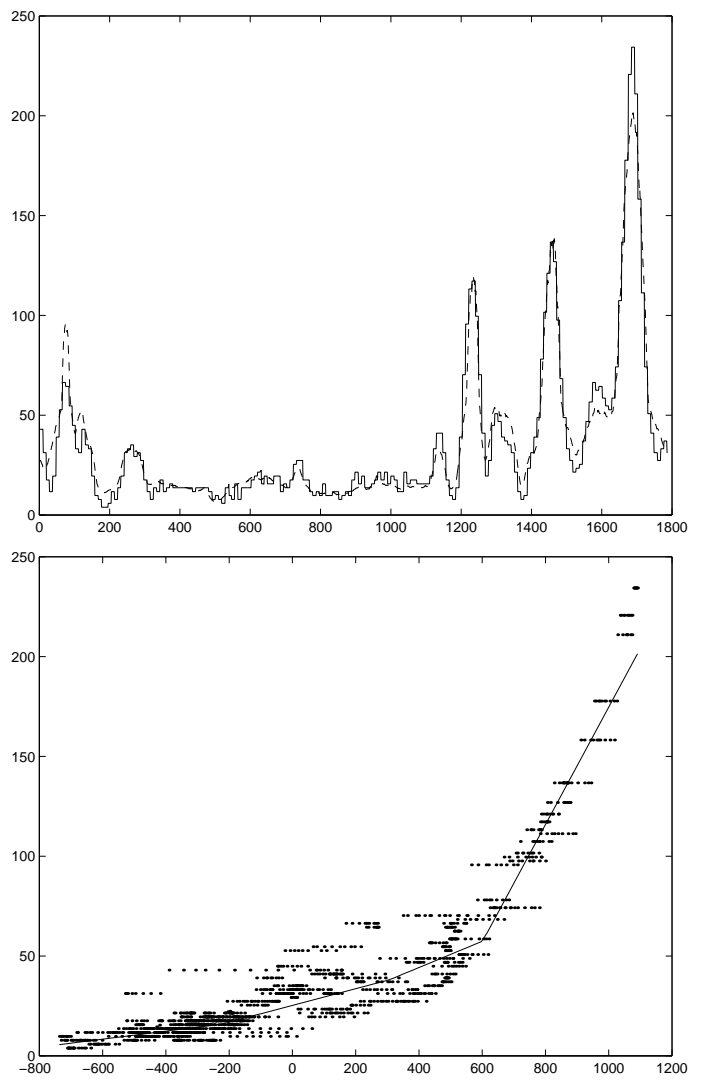

Figure 1: Simulation results using the maximum likelihood estimate of the model. Upper figure: Measured and estimated output. The solid line is the measured output, the dashed line the estimated output. Lower figure: The solid line is the estimated nonlinearity, the dots represent the estimated $x$ versus the measured $y$.

fication using separable least-squares. Submitted to European Journal of Control, December 1997.

[2] J. E. Dennis Jr and R. B. Schnabel. Numerical Methods for Unconstrained Optimization and Nonlinear Equations. Prentice-Hall, Englewood Cliffs, NJ, 1983.

[3] A. D. Kalafatis, L. Wang, and W. R. Cluett. Identification of wiener-type nonlinear systems in a noisy environment. Int J Control, 66(6):923-941, 1997.

[4] Lennart Ljung. System Identification, Theory for the User. Prentice Hall, 1987.

[5] G. A. Pajunen. Adaptive control of wiener type nonlinear systems. Automatica, 28:781-785, 1992.

[6] P. Pucar and J. Sjöberg. On the parametrization of hinging hyperplanes models. Technical report.

[7] Torbjörn Wigren. Recursive prediction error identification using the nonlinear wiener model. $A u$ tomatica, 29(4):1011-1025, 1993. 